ISSN 1112-9867

Available online at

http://www.jfas.info

\title{
USE OF GEOPHYSICAL METHODS FOR THE DEMONSTRATION OF KARSTIC PHENOMENA IN SOUTHERN ALGERIA: \\ GUERRARA AREA (DISTRICT OF GHARDAÏA. ALGERIA)
}

\author{
O. Melouah*, A. Zeddouri
}

Université Kasdi Merbah, Laboratoire des réservoirs souterrains Pétroliers, Gaziers et Aquifères, BP 511, Route Ghardaïa, Ouargla, Algérie

Received: 29 June 2016 / Accepted: 30 August 2016 / Published online: 01 September 2016

\begin{abstract}
Karst and pseudo-karst result from natural phenomena associated with the circulation of water through soluble geological environments, causing subsidence, sinkholes, cavities and collapses. During the last five years, Guerrara area (southern Algeria) was the subject of multidisciplinary studies to characterize the geological formations and assess the impact of their distribution on the movement of water surface and deep aquifers.

This study undertaken in three sites from the Guerrara area aims at the recognition and the characterization of underground voids (size, extension) by geophysical methods such as vertical electrical soundings and electrical tomography combined with $2 \mathrm{D}$ electromagnetic imaging ground penetrating radar GPR.
\end{abstract}

KEY WORDS: Pseudo-karst, sinkholes, Collapses, Ground penetrating radar, Cavities.

Author Correspondence, e-mail: oualid411@yahoo.fr

doi: http://dx.doi.org/10.4314/jfas.v8i3.24

\section{INTRODUCTION}

Les méthodes géophysiques sont un moyen efficace de détection de phénomènes karstiques sous couverture [1] et sont particulièrement utiles en milieu urbain pour Prévenir les risques 
naturels [2].

Le phénomène clef de la karstification est la dissolution de la roche par le $\mathrm{CO}_{2}$ dissous ayant plusieurs origines dans les eaux karstiques. Il provient principalement de la respiration racinaire et de la dégradation de la matière organique du sol par l'activité biologique et bactériologique, D'autres facteurs interviennent dans la dissolution des roches carbonatées comme la lithologie, la texture du sol et la fracturation.

Plusieurs travaux dans le monde en mis en évidence la présence des phénomènes karstiques dans les zones arides est semi arides et l'apparition de géomorphologie spécifiques tell que les Dayas, ces formations ont comme origine la dissolution des sels et des carbonates [3] par les eaux chargées en acide carbonique.

Dans le Nord du Sahara algérien le phénomène a été décrit par le Colonel Flatters durant ses missions de reconnaissance, plusieurs dépressions sont visible a partir des photos aériennes et satellitaires traduisant un phénomène a ampleur régionale.

Les sites choisis pour l'étude sont situés aux alentours de la région de Guerrara à quelques 120 $\mathrm{Km}$ au Nord-Est de la ville de Ghardaïa, et au sud de la région de Djelfa.

L'objectif visé a travers cette étude est la caractérisation géophysique et géotechnique des effondrements liée à la karstification et l'identification précoce du phénomène de part et d'autre de la ville de Guerrara afin de minimiser les risques sur les infrastructures et les vies humaines.

\subsection{Contexte geologique et hydrogeologique}

La géologie régionale est constituée en surface d'alluvions quaternaires, des grès du Mio-Pliocène et, dans certains endroits, de calcaire phosphaté de l'Eocène (fig.1, a). En profondeur l'alternance entre les calcaires, les argiles et les gypses constitue le reste du vestige sédimentaire de la région jusqu'à $650 \mathrm{~m}$ de profondeur.

Les potentialités hydrogéologiques sont représentées par la nappe du Mio-Pliocène et de l'Eocène à la surface avec une épaisseur de 200 m environ.

La nappe sénono-turonienne, constituée de formation carbonatée, est épaisse de $275 \mathrm{~m}$ environ. 
La nappe du Continental Intercalaire est constituée de sable, grès et argile avec un passage dolomitique attribué à l'Aptien. L'épaisseur moyenne de la nappe est de l'ordre de $650 \mathrm{~m}$ et le Toit varie entre 500 et $900 \mathrm{~m}$ de profondeur dans le sens Est-Ouest de la région de Guerrerra.

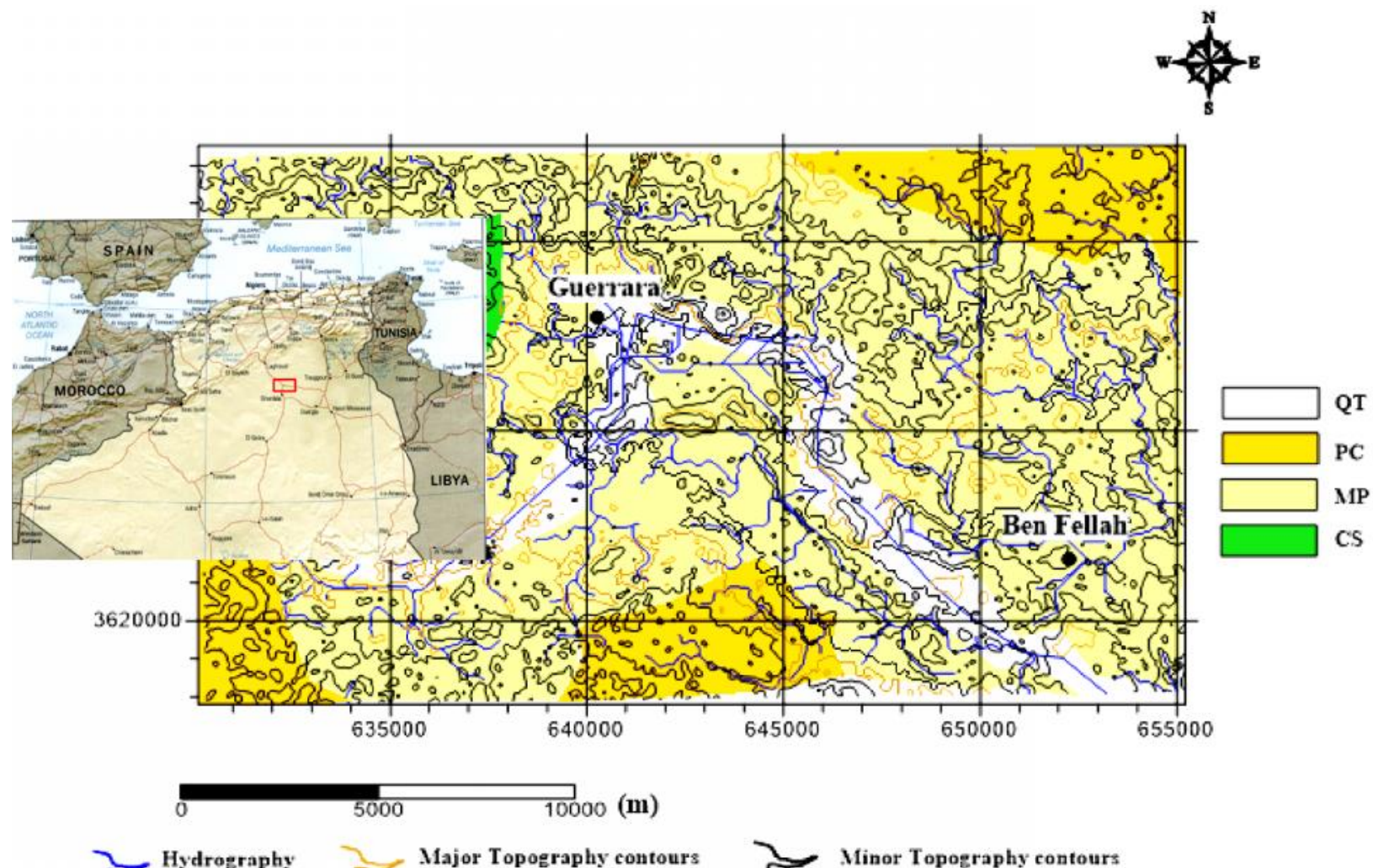

Elevation (m)

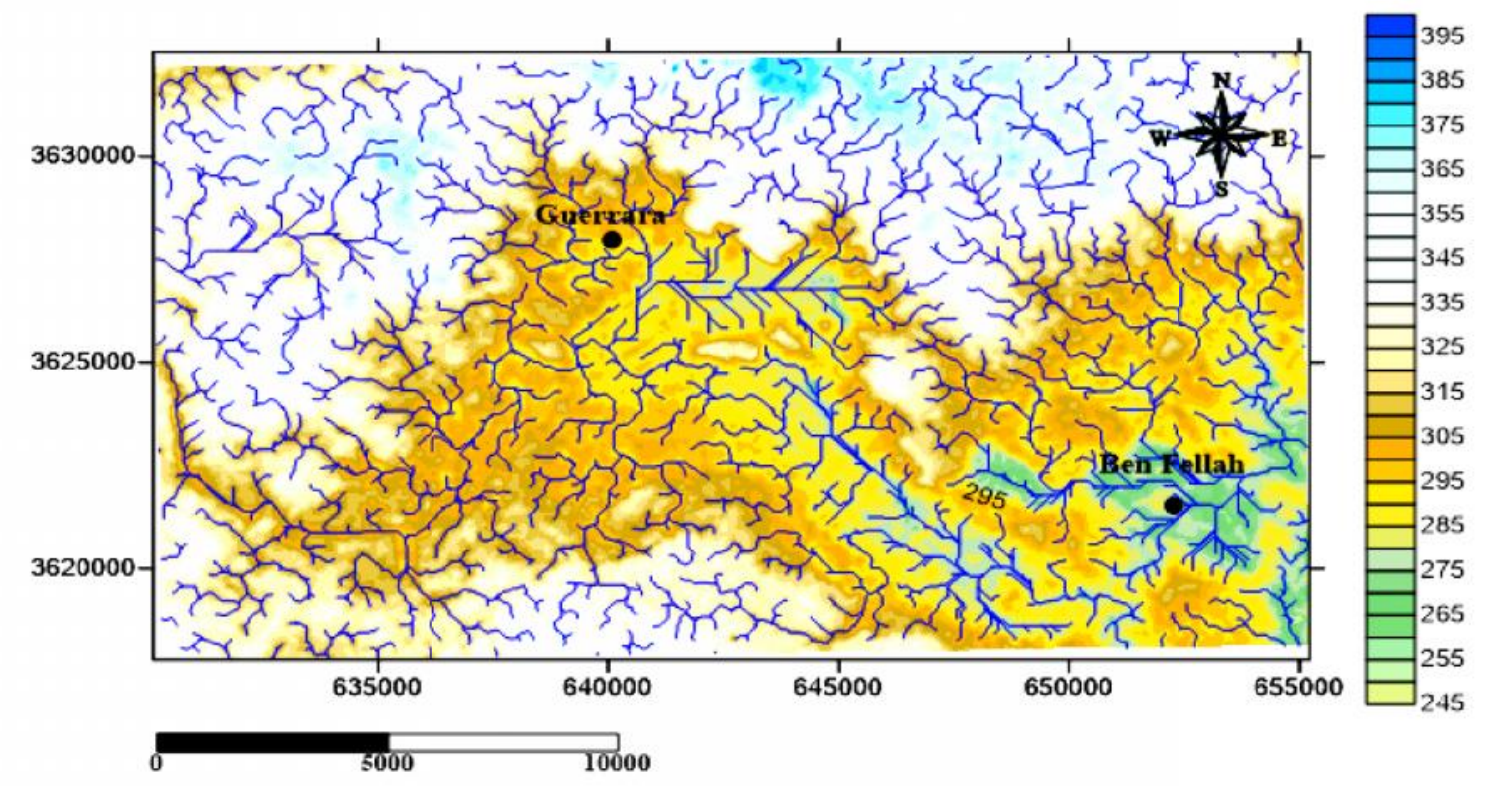

Fig.1. (a) carte géologique de la région de Guerrara: QT Quaternaire, PC Pliocène continental, MP Miopliocene, CS Crétacé supérieur, (b) carte topographique et réseau hydrographique de la région d'étude 


\section{RESULTS ET DISCUSSION}

Les compagnes d'acquisitions réalisées à travers les trois sites investigués ont permis d'obtenir les résultats suivants :

\section{2-1.Site 1 (palmeraie de guerrara)}

La réalisation des sondages électriques dans la zones de la palmeraie (fig2) a permis de classer les terrains rencontrés en quatre classes selon leurs résistivité : la première classe correspond à des matériaux ayant une résistivité électrique qui varie entre 40 et 200 .m ; la deuxième classe de résistivité correspond à des valeurs entre 600 et 800 .m ; la troisième gamme englobe des formations ayant des résistivités comprises entre 1100 et 2000 .m , la dernière classe montre des anomalies géo-électriques caractérisées par des résistivités supérieures à 3500 .m.

La corrélation entre les données des sondages électriques avec les données des forages a permis la mise en évidence de 04 anomalies à très fortes résistivités situées dans des formations de nature gréseuses à carbonatées (fig.3).Ces valeurs reliées à la présence des vides souterrains sont observées à partir des profondeurs qui varient entre 13 et $44 \mathrm{~m}$

Les classes de résistivités ayant des valeurs comprises entre 40 et $200 \mathrm{Ohm}$ m correspondent à des formations alluvionnaires englobant des dépôts limoneux sableux argileux et des grés altérés (formations d'âge quaternaires et Mio-pliocène) caractérisées par des faibles résistivités électriques (selon l'état de saturation en eau et la constitution du ciment gréseux ainsi que l'état de la roche soit seine ou fracturée, compacte ou décompressé).

L'interface géo-électrique caractérisée par des résistivités entre 600 à 800 Ohm.m correspond au toit des formations carbonatées de l'Eocène (à caractère continental) et des grés profonds compacts.Les résistivités entre 1100 et 2000 Ohm.m correspondent aux formations carbonatés Sénoniennes proprement dites à caractère marin. 


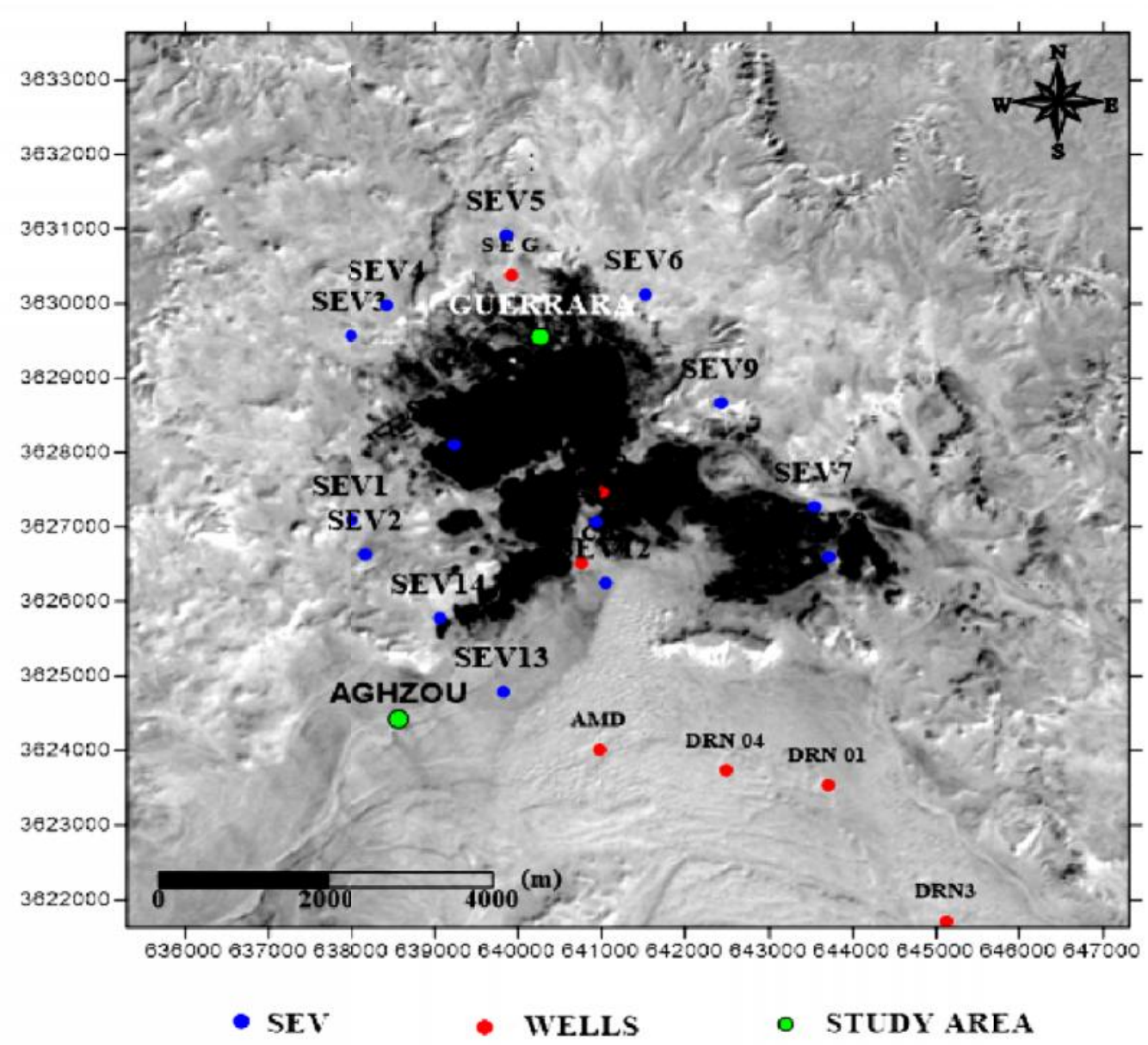

Fig. 2. Distribution des sondages électriques verticaux et des forages d'eaux dans la région 

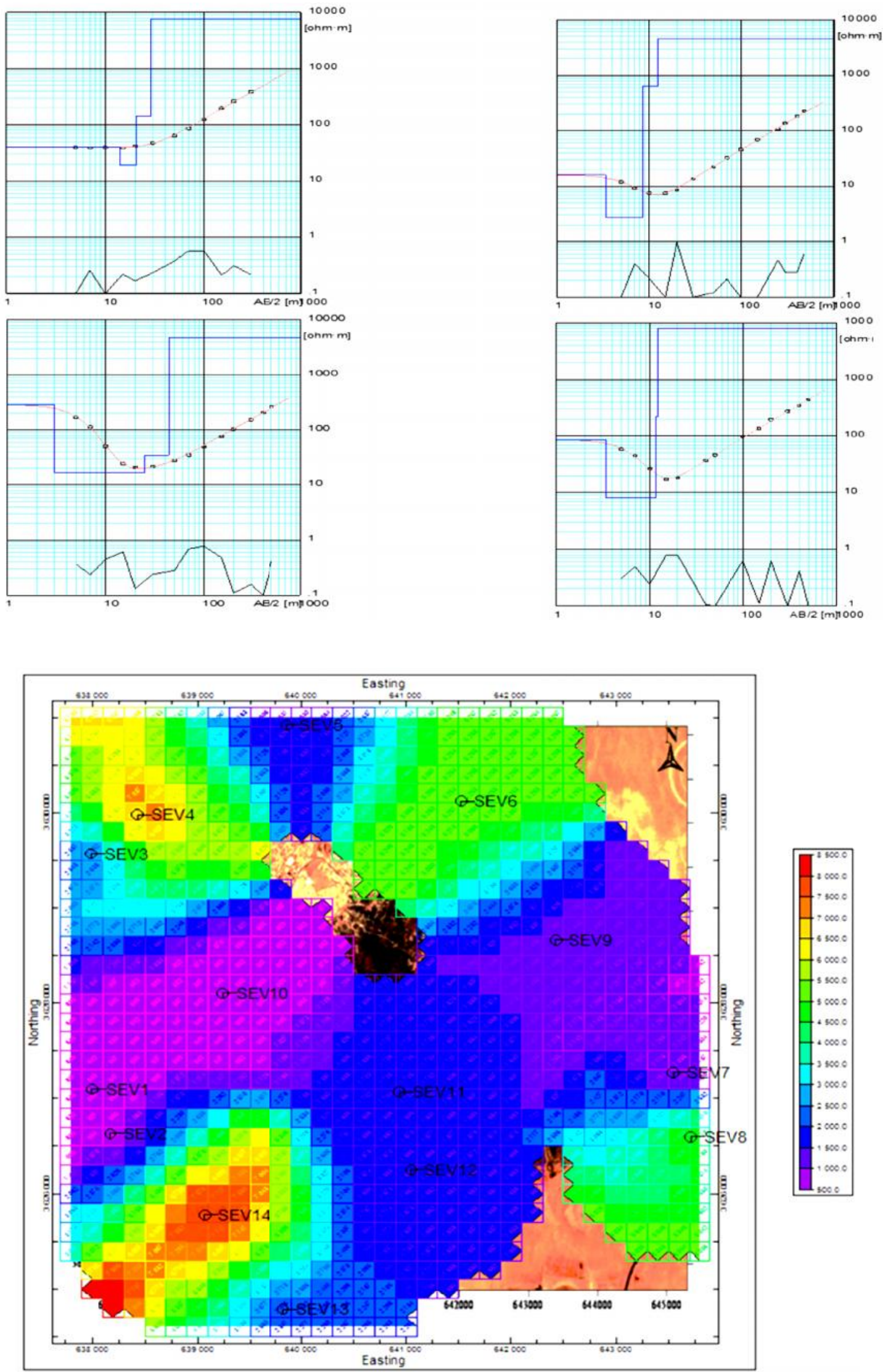

Fig.3. Résultats des sondages électriques site $\mathrm{N}^{\circ} 1$ 


\section{2-2.Site 2 (effondrement d'aghzou) :}

La pseudo-section1 de tomographie électrique indique la présence de deux anomalies dans la partie centrale et sud du profill à partir de $1.25 \mathrm{~m}$ de profondeur caractérisées par des résistivités élevées de l'ordre de 1400 Ohm.m, cela est interprété par la présence de cavité issue de la dissolution des formations alluvionnaires (formation de résistivité 270 à 600 Ohms.m), les grès sont caractérisés par des résistivités entre 80 et 250 Ohm.m et une épaisseur de 2 à $6 \mathrm{~m}$, les formations sableuse ont une résistivité de 35 à 85 Ohm.m et des épaisseurs variables.

Les mêmes formations sont observées dans les profils 2 et 3 de tomographie électriques avec une légère différence de quelques Ohm.m. Les cavités dans les profils 2et 3 ont des résistivités de 1000 et 3500 Ohm.m environ

Les profils Géoradar réalisé à proximité de l'effondrement ont permis de détecter plusieurs anomalies liées à la présence d'une formation géologique de nature conglomératique à la surface et de nature alluviono-gréseuse jusqu'à $5 \mathrm{~m}$ de profondeur. Les anomalies correspondant à la présence de vide se manifestent par des diffractions multiples du signal émis dues au contraste entre les matériaux géologiques et l'air ainsi que par la présence d'hyperbole de diffraction à très haute énergie.

Les essais géotechniques effectués sur les échantillons de la coupe AGZ ont révélé l'existence d'un potentiel de karstification hautement significatif. Les formations traversées par la coupe présentent des taux importants de gypse dépassant les $40 \%$ dans les alluvions et un taux de carbonates de $65 \%$ et $10 \%$ pour les conglomérats et les grés.

Les essais de compression sur les cubes de grés dimensionnés à $4 \times 4$ et $5 \times 5 \mathrm{~cm}$ ont révélé le caractère fragile et tendre de cette formation avec une valeur moyenne de la résistance à l'écrasement de $11 \mathrm{MPa}$.

\section{2-3. Site 3 (dayat ben fellah) :}

Les 10 profils de tomographie électriques réalisés dans le site de Dayat Ben Fellah (fig. 6) ont révélé la diversité géologique des terrains investigués, à l'intérieur de la cuvette, le bed rock gréseux est situé à moins de 10 m de profondeur avec des résistivités entre 80 et 140 . 

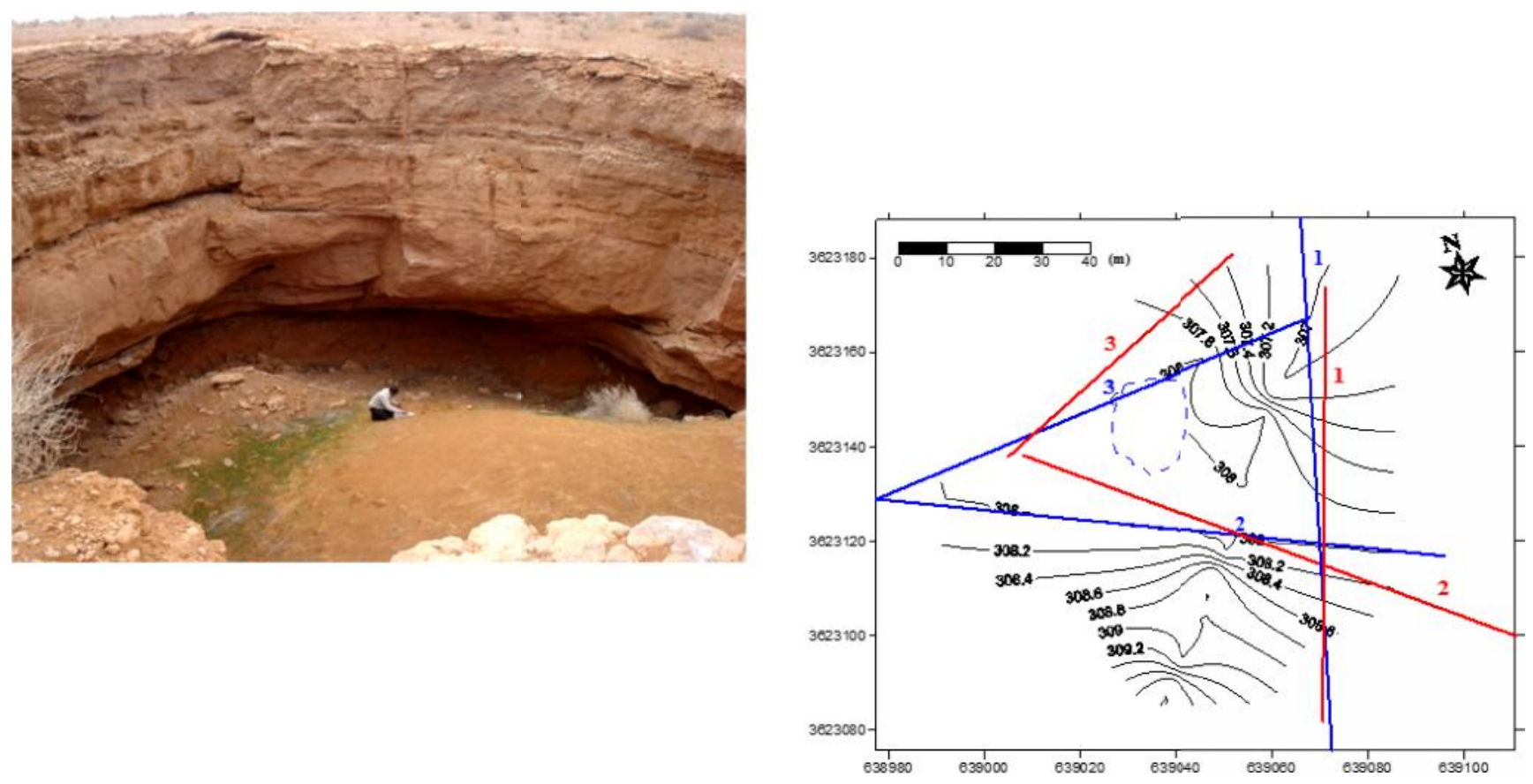

Fig.4. Site 2 effondrement de Aghzou, en rouge profils de radar géologique, en bleu profil de tomographie électriques 2D

\section{3-3. Site 3 (dayat ben fellah) :}

Les 10 profils de tomographie électriques réalisés dans le site de Dayat Ben Fellah (fig. 6) ont révélé la diversité géologique des terrains investigués, à l'intérieur de la cuvette, le bed rock gréseux est situé à moins de $10 \mathrm{~m}$ de profondeur avec des résistivités entre 80 et 140 Ohm.m. Les formations alluvionnaires sont caractérisées généralement par des faibles résistivités entre 20 et 70 Ohm.m. Les cavités observées sont soit comblées ou semi-comblées par des matériaux conducteurs, ce qui réduit partiellement le contraste géo-électrique entre l'encaissant rocheux et les vides détectés.

Dans les hauteurs de Dayat ben fellah, les données acquises ont mis en évidence plusieurs anomalies liées à l'activité tectonique se manifestant par la géométrie des terrains investigués et le contraste remarquable des résistivités.

Les poches de dissolution sont bien marqués dans l'ensemble de ces profils vue la nature géologique gypso-carbonatée des formations situées dans les hauteurs (fig. 7. a, b, c).

Les données du GPR ont confirmé la présence des observations indiquées par la tomographie électriques. Le bed rock gréseux est situé à des profondeurs variables, allant de $1 \mathrm{~m}$ à des profondeurs indéfinies. Cette ambiguïté est due au contexte géologique conducteur de la zone 

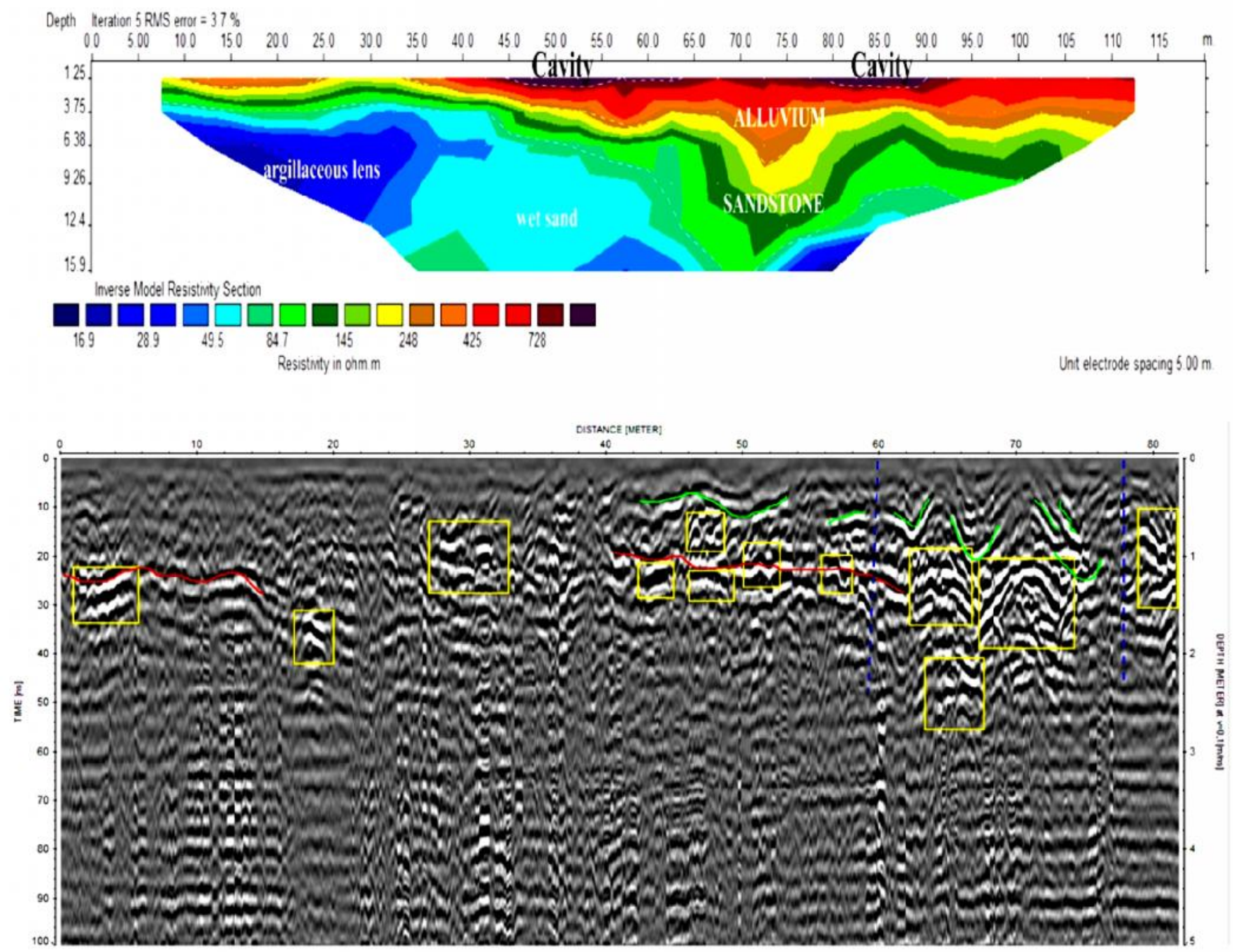

\section{Cavity}

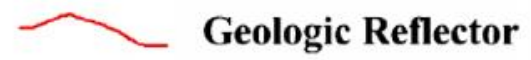

Tectonic Joint

Sinkhole Limits

Fig.5. a) résultats de la tomographie électriques profil $\mathrm{N}^{\circ} 1$ site 2 , b) radar géologique profil $\mathrm{N}^{\circ} 2$ effondrement d'Aghzou.

Ce qui réduit d'une par la profondeur d'investigation et d'autre part diminue la résolution des profils réalisés [4]. Malgré ces conditions plusieurs anomalies ont été détectées à partir de 3m de profondeur dans la cuvette de l'effondrement ayant la même signature électromagnétique que ceux détectés dans le site 2 correspondant à la présence de vides souterrains à dimensions métriques (fig. 7.d).

Par ailleurs, quatre coupes géologiques ont été réalisées sur ce site; parmi lesquelles une seule coupe (la coupe DBA) a été choisie pour faire le sujet d'essais géotechniques. Le choix de cette coupe se justifie par l'accessibilité à l'endroit d'une part et par l'homogénéité spatiale 
des formations sur toute la superficie du vestige.

Les formations superficielles sont constituées par des grés argileux et carbonatés et des produits d'altération rubéfiés. La continuité de la coupe est composée de produit d'alluvionnement consolidé à granulats moyen à fin et à microcavités et alvéoles.

Les résultats des essais chimiques se concordent avec les données géo-électriques obtenues car celles-ci ont révélé la présence d'un taux important de carbonates dépassants les 35\% traduisant la grande solubilité des formations analysées. Les essais de résistance à la compression réalisés sur les formations échantillonnées présentent des résultats variables mais confirmant tout de même leur caractère fragile et tendre surtout dans les formations alluvionnaires (Compression strength=19 bars).

En général, les alluvions rencontrées à partir de $1 \mathrm{~m}$ de profondeur ont tendance à être tendres et altérés. Ces résultats sont en étroite relation avec les conditions et l'intensité des facteurs d'altération et d'érosion dans la zone. Les observations faites durant l'échantillonnage de ces matériaux confirment les résultats de nos testes à savoir la présence des microcavités et des alvéoles.

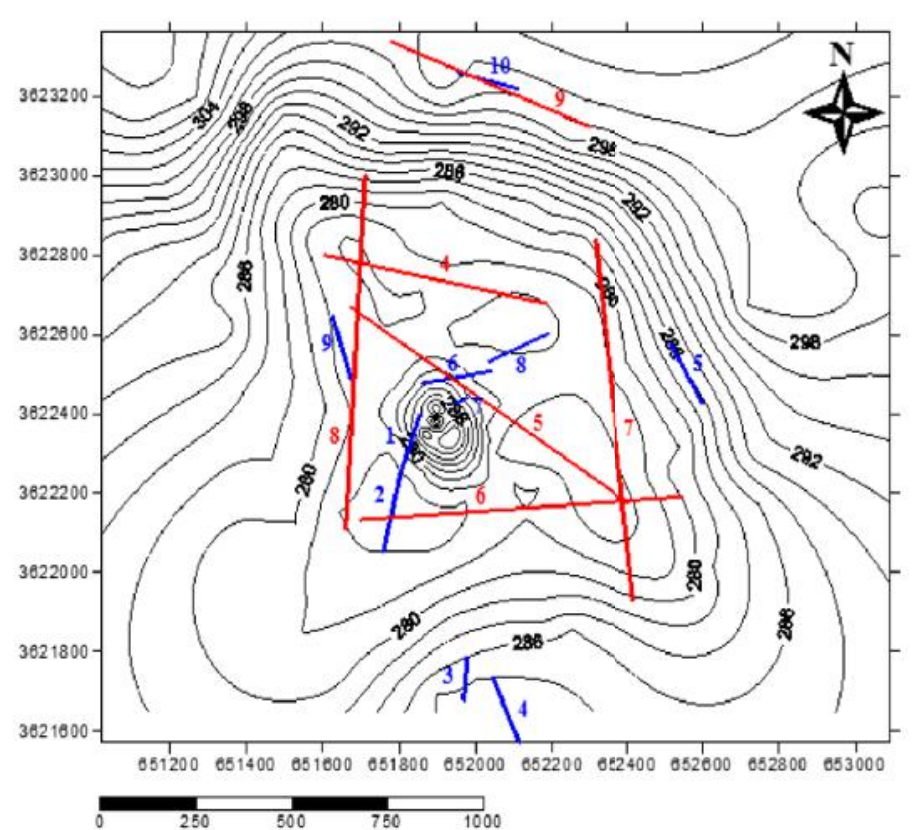

- Profiles de Tomographie de Résistivité électriques 2D
Profiles de Radar géologique

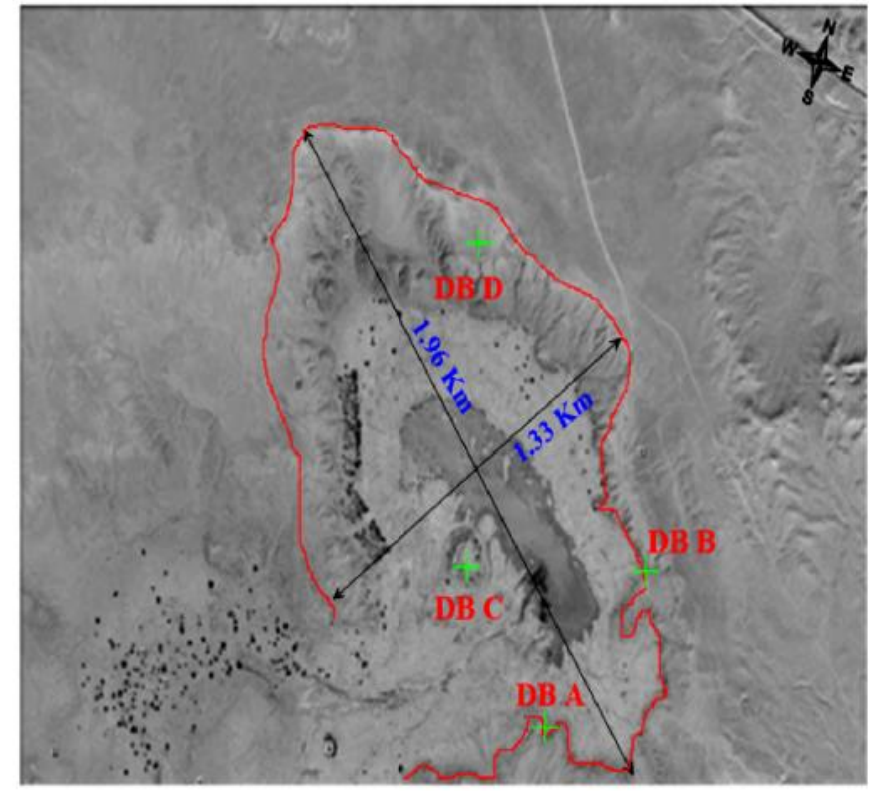

\section{Cross Section}

Fig.6. Site 3 Dayat Ben Fellah, en rouge profile de radar géologique, en bleu profil de tomographie de résistivités électriques 2D. 


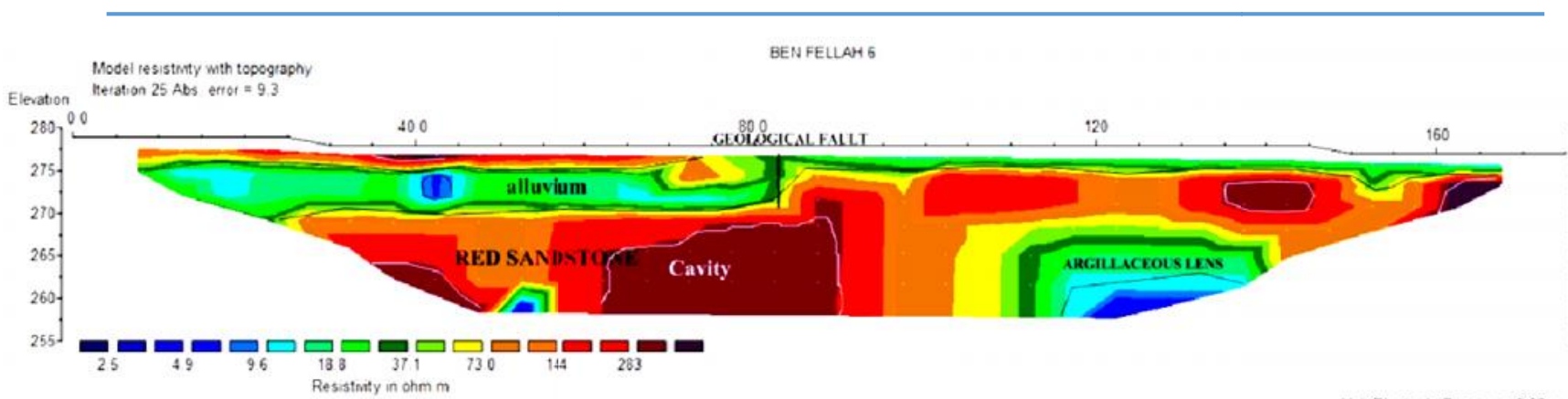

BEN FELLAH 2

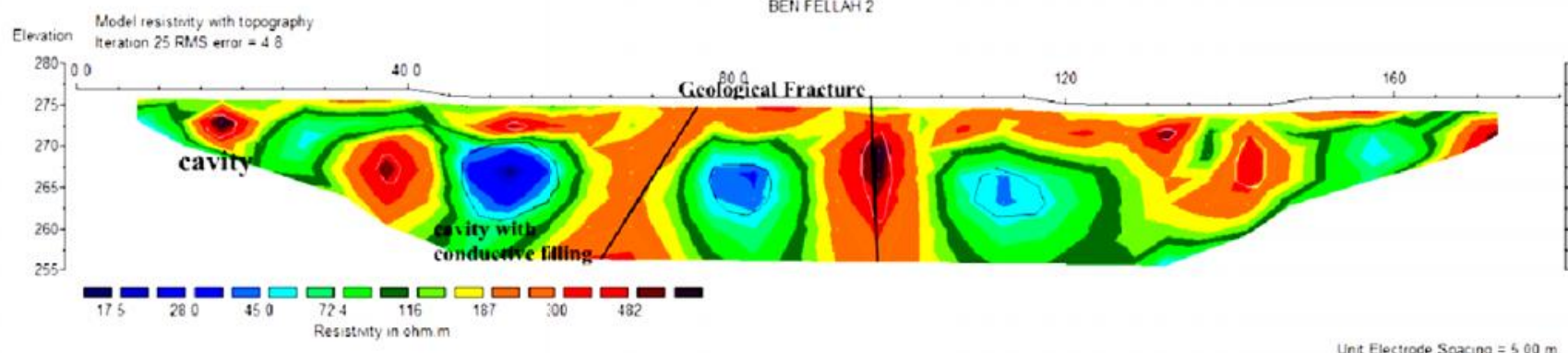

BEN FELLAH 10
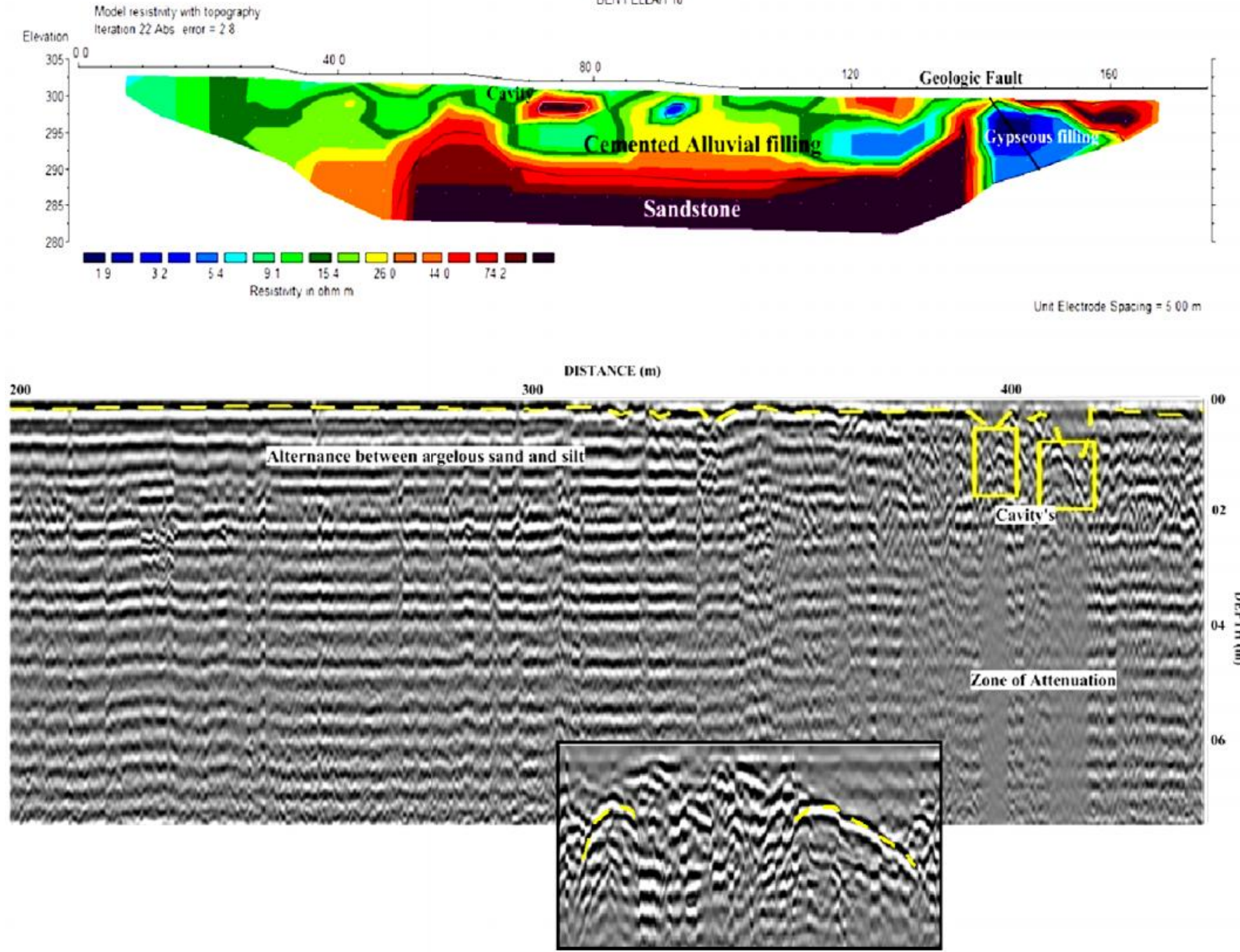

Fig. 7. a),b),c ) résultats de l'inversion des profil $\mathrm{N}^{\circ} 6,2,10$ de tomographie électrique dans le site de Ben fellah site, $\mathbf{d}$ ) interprétation des résultats du radar géologique profil $\mathrm{N}^{\circ} 5$ 
Dans les sites d'El Amied-Drine-Guerrara, le modèle tridimensionnel issu des données des sondages électriques verticaux a permis d'avoir une idée de la distribution des résistivités électriques dans le sous-sol. La corrélation de ces données avec les forages préexistants a permis de différencier quatre unités géo-électriques : les sédiments alluvionnaires de surface, les grès compacts, les calcaires sénoniens marins et les anomalies dues à la présence de cavités proches de la surface avec des résistivités dépassant $3500 \quad$.m.

Quatre cavités, essentiellement dans les formations gréseuses et carbonatées, sont situées dans les hauteurs de Guerrara au Nord et dans les extrémités Sud et Est de la ville de Guerrara.

Au niveau du site d'Aghzou, les visites sur terrains menées au voisinage de l'effondrement a permis de localiser un nombre important de cavités situées entre 1 et $2 \mathrm{~m}$ de profondeur dans les formations alluvionnaires gypseuses. Les formations gréseuses situées en dessous sont à leur tour constituées par des sables consolidés par un ciment carbonatée, fracturés et caractérisés par une faible résistance à la compression.

Ces fractures observées en surface et détectées par les diverses méthodes d'imagerie sont à l'origine de l'effondrement. Sous l'effet de l'alluvionnement, les eaux agressives chargées en acide carbonique entrent en réaction avec les constituants solubles des formations alluvionnaires (gypse et carbonates) créant ainsi des zones de passage préférentielle pour s'infiltrer par la suite à travers les grés à ciment carbonaté fracturé et tendre pour créer des cavités de dimensions variables engendrant la perte de portance et ainsi l'effondrement.

L'ensemble des cavités détectées par les méthodes géophysiques sont situées dans le Sud-Ouest de l'effondrement, supposé être le futur front de l'effondrement.

Au niveau du site de Dayat Ben Fellah, l'investigation menée par les techniques géophysiques a mis en évidence la présence de plusieurs structures cavitaires situées dans la cuvette de l'effondrement et dans les hauteurs périphériques ces vestiges sont généralement de dimensions métriques.

Les cavités observés semblent être orientées suivant les directions $\mathrm{N} 50-70^{\circ}$ et $\mathrm{N} 150$ $170^{\circ}$.Ces observations sont concordantes avec les orientations des événements tectoniques identifié sur site.

Les disparitions brutales des eaux alluviales de Dayat Ben Fellah peuvent être attribuées à la 
présence et la dispersion des cavités détectées. Celles-ci sont caractérisées par des dimensions métriques, et ayant des extensions verticales inconnues.

l'origine de la Dayat est due à un effondrement karstiques dans les formations carbonatées de l'Éocène. Aux terme des investigations réalisés, l'origine de l'effondrement est fortement lié à l'activité tectonique qui a créé des couloirs préférentielles d'érosion à travers les alluvions carbonatées situées jusqu'à des profondeurs dépassants les $10 \mathrm{~m}$. Ces chemins ont était façonnés par l'activité érosive des cours d'eau existant provocant la décalcification issue du potentiel chimique hautement agressive des eaux pluviales en contact avec les sols limoneux sableux argileux riches en acide humique .

Les cavités détectées sont non-connectées malgré leurs dimensions importantes, ce qui mène à penser à la présence d'un réseau karstique peu développé ou partiellement comblé par un volume important de sédiments apportés par les cours d'eau. Ces conclusions sont basées sur les résultats de l'imagerie géophysique obtenus qui démontrent la présence de lentilles conductrices dans la majorité des profils réalisés.

\section{MATÉRIELS ET MÉTHODES}

\section{3-1.Methodologie}

La caractérisation des phénomènes karstiques dans les zones arides nécessite un choix judicieux des techniques employé, la résistivité électriques et le Georadar sont les plus utilisé pour la détection des vides souterrains [5];[6];[7];[8]. Ces méthodes sont rapides non destructives et à coût réduit et fournissent des informations importantes sur les propriétés des formations de la sub-surface [9] ; [10].

En vue d'atteindre cet objectif, ont a fait recours à la combinaison des méthodes électriques représentées par les sondages électriques et la tomographie de résistivités 2D [1] ainsi que la méthode électromagnétique par le Géoradar à basse fréquence [11].

La diversité des techniques utilisées a permis d'atteindre des profondeurs variables dans les terrains investigués. Les sondages électriques verticaux par le dispositif Wenner-Schlumberger ont atteint $160 \mathrm{~m}$ de profondeur environ.

La technique TRE (tomographie de résistivité électrique) a permis d'atteindre des profondeurs 
moyennes de 20 m environ.

L'outil Georadar (de marque GSSI) basé sur la propagation des ondes électromagnétiques à hautes fréquence [12] a contribué à la détection des cavités ayant une signature électromagnétique bien marquée par rapport à l'encaissant géologique. La profondeur d'investigation atteinte est comprise entre 5 et $22 \mathrm{~m}$ en utilisant les fréquences de 200 et 100 MHZ.

L'utilisation des méthodes géotechniques classiques, a permis de préciser la nature des réactions entre la roche et les eaux y circulant et la vulnérabilité du paysage rocheux vis-à-vis le développement des systèmes karstiques et leurs extensions.

\section{3-2. Acquisition et traitements des donnees}

L'équipement Géoradar de type GSSI est composé d'une antenne blindée de fréquence 100-200 MHZ et une unité d'enregistrement SIR 20. Les données acquise sous format DZT ont subi des traitements adéquat afin d'améliorer la qualité de l'enregistrement, les filtres appliqués sont : la correction statique, les corrections de gain, back ground removal et le filtre passbande [13].

Les mesures radar permettent d'obtenir les temps aller-retour entre l'antenne et les divers échos produits par la structure.

La transformation des temps de propagation en distance s'effectue en utilisant la constante diélectrique du matériau traversé. Les valeurs déterminées dans cette étude ont été obtenues Après le calage des temps de parcours sur un réflecteur géologique à distance connue avec certitude [13].

Les résultats sont représentés sous forme de radargramme, la distance horizontale parcourue et mesurée par un dispositif spécifique placé à chaque acquisition dans les antennes.

La tomographie électrique a 2D est basée sur la mesure de la différence du potentiel électrique entre deux électrodes de réception MN. Le courant est injecté à travers deux électrodes A B, les dispositifs utilisés sont en fonction de 1'objectif à atteindre.

Les quadripôles Wenner présentent une bonne résolution verticale par contre les dispositifs Dipôle-Dipôle et Pole-Dipôle présentent une bonne résolution horizontale ce qui favorise leur utilisation dans la détection des structures verticales [14] ; [15]. 
Le Wenner- Schlumberger est un dispositif Hybride qui offre à la fois une bonne résolution horizontal et vertical [16]; [17].

Les donnes de tomographie électrique 2D et les Sondages électriques ont était acquise par un résistivimétre ABEM et (04) bobine de câble électrique chaque une de $500 \mathrm{~m}$ de linaire et une série de 37 électrodes, le dispositif choisi et le Wenner $\alpha$.

Les séries de mesures ont était effectué suivant un pas d'avancement fixe de $5 \mathrm{~m}$, les espaces inter électrodes utilisée sont 5, 10, 20, 30 et 40 m, les profondeurs d'investigations visées par ces arrangements sont de l'ordre de 2.5, 5, 10, 15 et $20 \mathrm{~m}$.

Les méthodes d'inversion choisis sont le square inversion et l'inversion robuste [18] .Afin de s'assurer de la fiabilité des résultats des corrections topographiques ont était induites aux modèles obtenus [19], les informations sur l'erreur et la longueur des profils réalisés sont représentés dans le tableau 1.

Pour les sondages électriques l'espacement entre les pôles d'injection AB est de 1000 ml, Le dispositif Wenner -Schlumberger a était calé en corrélant les résistivités électriques avec les données de forage profond réalisé auparavant, Les donnes acquise ont était inversé par le baie du logiciel winsev6

Des échantillons solide de roche et de sol au nombre de 35 ont était prélevé et traité afin de Déterminé les caractéristiques physique et chimique des terrains géologiques avoisinant les effondrements.

Généralement la zone et connue par ce type de désordre détecté surtout durant les travaux de forage et dans les zones urbaines qui ce manifeste par des pertes importantes de boue a partir de 60 a 220 m environ et des affaissement des constructions en sub surface, Durant les années 2007 jusqu'a 2012 la récolte des donnes géologiques et géophysique a permis de développé plusieurs hypothèses sur les modalités de la karstifications et des mécanismes d'effondrements dans la zone . 
Tableau 1: caractéristiques des profils de tomographie réalisez dans la région de Guérrara

\begin{tabular}{clcc}
\hline StudyArea & Profils & $\begin{array}{c}\text { \%RMS error } \\
\text { Resestivity model }\end{array}$ & $\begin{array}{c}\text { Length } \\
(\mathbf{m})\end{array}$ \\
\hline \multirow{4}{*}{ Aghzou } & Pr1 & 3,7 & 120 \\
& Pr2 & 5,2 & 120 \\
& Pr3 & 1,97 & 90 \\
\hline \multirow{5}{*}{ Ben Fellah } & Db1 & 1,6 & 170 \\
& Db2 & 4,8 & 180 \\
& Db3 & 3,9 & 110 \\
& Db6 & 4,8 & 180 \\
& Db7 & 4,8 & 175 \\
& Db8 & 9,3 & 175 \\
& Db9 & 3,3 & 45 \\
& Db10 & 2,9 & 175 \\
& 2,6 & 170 \\
\hline
\end{tabular}

\section{CONCLUSION}

Les cavités récences par les moyen géophysiques de sub-surface représente une petite partie du système karstique dans la région, l'étude de la fracturation est un moyen clés pour la compréhension de la genèse et du développement du karst, ces résultats préliminaires indique le développement de ces phénomènes dans les carbonates du sénonien et de l'éocène, l'analyses des logs de forages et l'utilisation des traceurs apporte plus d'information sur la géométrie du vestige.

\section{REMERCIMENTS}

Au titre de ce travail je tien a remercié le directeur du Laboratoire des réservoirs souterrains Pétroliers, Gaziers et Aquifères, ainsi que le responsable du centre de recherche scientifique de l'université Kasdi Merbah Ouargla. 


\section{REFERENCES}

[1] Coskun N. The effectiveness of electrical resistivity imaging in sinkhole investigations. I. J Phys.Scie., 2012, 7 (15), 2398 - 2405.

[2] El Khammari K, Najine A, Jaffal M. Aïfa T, Himi M, Vásquez D, Casas A, Andrieux P. Imagerie combinée géo-élèctrique-radar géologique des cavités Souterraines de la ville de Zaouit Ech Cheikh (Maroc). C. R. Géosc., 2007, 339(7), 460-467.

[3] Salomon J N. Les influences climatiques sur la Géomorphologie Karstique : Exemple des milieux tropicaux et arides. Quat., 1997, 8(2-3), 107-117.

[4] Annan A P, Davis J L. Impulse radar sounding in permafrost. Rad.Scie., 1976, 11(4), 383-394. DOI: 10.1029/RS011i004p00383.

[5] Beres M, Luetscher M, Olivier R. Integration of ground-penetrating radar and microgravimetric methods to map shallow caves. J.Appl .Geophy., 2001,46(4), 249-262. DOI: http://dx.DOI.org/10.1016/S0926-9851(01)00042-8.

[6] El-Awadi E, El-Qady G, Nigm A, Shaaban F, Ushijima K. Integrated Geophysical Survey for Site Investigation at a New Dwelling Area, Egypt .J.Envir.Engin.Geophy., 2006,11(04), 249-259.

[7] Xu X, Zeng Q, Li D, Wu J, Wu X, Shen J . GPR detection of several common subsurface voids inside dikes and dams. Engin. Geolo., 2010, 111(1-4), 31-42. DOI:10.1016/j.enggeo.2009.12.001.

[8] Vadillo I, Benavente J, Neukum C, Grützner C, Carrasco F, Azzam R, Liñán C, Reicherter K. Surface geophysics and borehole inspection as an aid to characterizing karst voids and vadose ventilation patterns (Nerja research site, S. Spain). J. Appl.Geophy., 2012, 82,153-162. DOI:10.1016/j.jappgeo.2012.03.006.

[9] Sasaki Y. Resolution of resistivity tomography inferred from numerical simulation. Geophy. Prosp., 1992, 40(4), 453-463. DOI: 10.1111/j.1365-2478.1992.tb00536.x.

[10] Store H, Storz W, Jacobs F. Electrical resistivity tomography to investigate geological structures of earth's upper crust. Geophy .Prosp., 2000, 48(3), 455-471.

[11] Porsani J L. GPR survey at Lapa do Santo archaeological site, Lagoa Santa karstic region, Minas Gerais state, Brazil .J .Archa .Scie., 2010,37(6) ,1141-1148. 
DOI: $10.1016 /$ j.jas.2009.12.028.

[12] Davis J L, Annan A P. Ground-penetrating radar for high-resolution mapping of soil and rock stratigraphy. Geophy.Prosp., 1989, 37(5), 531-551. DOI: 10.1111/j.1365-2478.1989.tb02221.x.

[13] Bano M, Marquis G, Nivière B, Maurin J C, Cushing M. Investigating alluvial and tectonic features with ground-penetrating radar and analyzing diffractions patterns; J. Appl.Geophy., 2000, 43(1), 33-41.

[14] Ward S H 1989. Resistivity and induced polarization methods. In geotechnical and environmental geophysics.S.Expl.Geophy.1989,1,147-190.DOI:10.1190/1.9781560802785.ch 6.

[15] Stummer P, Maurer H, Green, A. Experimental design, Electrical resistivity data sets that provide optimum subsurface information. Geophy., 2004, 69(1), 120-139. DOI: $10.1190 / 1.1649381$.

[16] Marescot L, Loke M H, Chapellier D, Delaloye R, Lambiel C, Reynard E. Assessing reliability of 2D resistivity imaging in mountain permafrost studies using. The depth of investigation index method. N. Surf. Geophy., 2003,1(2), 57-67. DOI: 10.3997/1873-0604.2002007.

[17] Dahlin T, Zhou B. A numerical comparison of 2D resistivity imaging with 10 electrode arrays. Geophy. Prosp., 2004, 52(5), 379-398. DOI: 10.1111/j.1365-2478.2004.00423.x.

[18] Loke M H, Barker R D. Rapid least-squares inversion of apparent resistivity pseudo sections using a quasi-Newton method. Geophy. Prosp., 1996, 44(1), 131-152. DOI: 10.1111/j.1365-2478.1996.tb00142.x.

[19] Scapozza, C, Lambiel C, Baron L , Marescot L, Reynard E. Internal structure and permafrost distribution in two alpine periglacial talus slopes, Valais, Swiss Alps. Geom., 2011, 132(3-4), 208-221. DOI:10.1016/j.geomorph.2011.05.010.

\section{How to cite this article:}

Melouah O, Zeddouri A.Use of geophysical methods for the demonstration of karstic phenomena in southern Algeria: guerrara area (district of ghardaïa. algeria). J. Fundam. Appl. Sci., 2016, 8(3), 1097-1114. 